\title{
Reversal of new oral anticoagulants and personalized medicine
}

\begin{abstract}
"Given that innovation is a way of life, researchers constantly strive to develop new anticoagulants and their antagonists, sometimes being unaware of their necessity, effectiveness and cost."
\end{abstract}

First draft submitted: 6 October 2016; Accepted for publication: 20 October 2016; Published online: 7 December 2016

Keywords: antagonists $\bullet$ anticoagulant $\bullet$ antiplatelet $\bullet$ bleeding $\bullet$ fibrinolytic

- hypercoagulant $\bullet$ hyper-reactivity $\bullet$ pharmacokinetics $\bullet$ procoagulant $\bullet$ thrombosis

Given that innovation is a way of life, researchers constantly strive to develop new anticoagulants and their antagonists, sometimes being unaware of their necessity, effectiveness and cost. Scientists are fascinated by new anticoagulants and their reversal agents. Despite their enormous costs and difficulty in monitoring, we somehow tend to prefer the new oral anticoagulant drugs just because they are new and have been found to be noninferior over warfarin in clinical trials.

Unfractionated heparin, a parenteral anticoagulant, and warfarin, an oral anticoagulant, have been conventionally used as anticoagulants. Heparin is effectively monitored by activated partial thromboplastin time (APTT) and the success of heparin use lies in the availability of protamine sulfate as an effective antidote. Warfarin, on the other hand, is effectively monitored by prothrombin time/international normalized ratio (PT/INR) and relies on the availability of vitamin $\mathrm{K}$ as its most effective antagonist. There have been newer strides with the development and approval of low-molecularweight heparins but so far there are no effective specific antagonists, but rather there is reliance on protamine sulfate which is only partially effective.

\section{Nonvitamin k antagonist oral anticoagulants}

As we commemorate 100 years of the use of heparin and more than 50 years of use of warfarin, four new nonvitamin $\mathrm{K}$ antagonist oral anticoagulants (NOACs) have been approved by the US FDA since 2010. One among them is a thrombin inhibitor (dabigatran) while the other three are factor $\mathrm{Xa}$ inhibitors (rivaroxaban, apixaban and edoxaban). A fourth factor Xa inhibitor, betrixaban, which has a lower renal clearance and hepatic metabolism and a longer half-life compared with approved NOACs, is in clinical development $[1,2]$. Although NOACs have decreased bleeding risk with noninferior efficacy when compared with warfarin in patients with atrial fibrillation [3-7], an effective and specific antagonist is still absolutely necessary. While nonspecific hemostatic agents such as prothrombin complex concentrates (PCCs), activated PCCs and recombinant factor VIIa may be used, more specific options are desirable. One such specific agent is the approved humanized monoclonal antibody fragment, idarucizumab, which is used as a reversal agent for dabigatran. The investigational factor $\mathrm{Xa}$ decoy andexanet alfa and the synthetic, small molecule, ciraparantag, may reverse the anticoagulant effects of factor Xa inhibitors.

All anticoagulants cause bleeding. The success of any anticoagulant besides its safety and efficacy is dependent on the availability of a specific, safe and effective antagonist. Heparin and warfarin serve as examples whose anticoagulant effects can be easily reversed. During scenarios such as serious

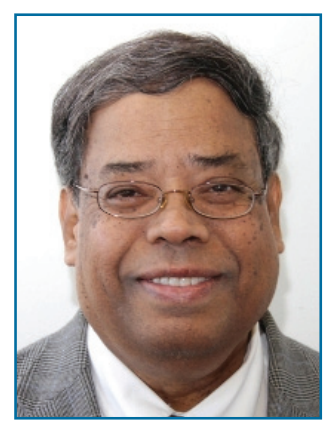

Omer lqbal

Author for correspondence: Department Pathology \& Ophthalmology, Loyola University Medical Center, Maywood, IL 60153, USA oiqbal@luc.edu

Walter Jeske Department of Thoracic \& Cardiovascular Surgery \& Pathology, Loyola University Medical Center, Maywood, IL 60153, USA

Jawed Fareed Department Pathology \& Pharmacology, Loyola University Medical Center, Maywood, IL 60153, USA

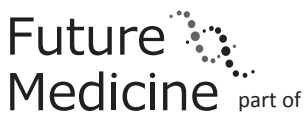


bleeding or the need for emergency surgery, a physician needs to know the circulating concentration of the drug or the residual anticoagulant activity. For dabigatran, given that most institutions are not adept at measuring the serum concentration of dabigatran, one has to be content with tests such as the activated partial thromboplastin time or the too sensitive dilute thrombin time and the generally unavailable ecarin clotting time tests. In the case of factor Xa inhibitors, physicians must rely on highly variable prothrombin time or the too sensitive and often clinically unavailable anti-Xa assays. The recommended chromogenic anti-Xa assays still need validation and have a delayed turnaround time, making them impractical in emergency settings.

\section{Reversing the effects of NOACs}

The areas of uncertainty in the management of NOACs are perioperative management, laboratory monitoring of anticoagulation and management of bleeding complications. During the management of periprocedural anticoagulation with NOACs, both the risks of bleeding and thrombosis should be evaluated and the decision to cease the NOAC should be individualized or personalized [7]. Due to a lack of specific tests, the monitoring issues in assessing the residual activity become compounded both before and after using either the nonspecific hemostatic agents or the specific NOAC reversal agents. The nonspecific hemostatic strategies include alteration of pharmacokinetics of NOACs, antifibrinolytic drugs, PCCs, activated PCCs, factor eight bypassing activity, recombinant factor VIIa, cryoprecipitates and fresh frozen plasma. While fresh frozen plasma should not be used to reverse the anticoagulant effects of NOACs because of its limited effectiveness by virtue of lower concentrations of clotting factors and the potential for heart failure due to volume overload [8], the other nonspecific agents are equally impractical due to their variable efficacy $[9,10]$. While the absorption of dabigatran can be reduced by the administration of activated charcoal, the absorption of rivaroxaban is unaffected. It is unclear whether the absorption of apixaban and edoxaban can be modified by the administration of activated charcoal. Dabigatran, due to its low binding of plasma proteins, could be eliminated from the circulation by performing hemodialysis. Since factor Xa inhibitors have higher binding to plasma protein fractions, they are not effectively eliminated by hemodialysis. With regard to the specific reversal agents, Idarucizumab was initially granted priority review by the FDA in order to accelerate its approval. Following its approval in October 2015, there have been at least four critically ill patients with dabigatran-induced bleeding who failed to reach effective hemostasis with idarucizumab [11-14]. In addition to these four cases, the real world data will eventually prove the degree of validity of idarucizumab as an antidote for dabigatran. Andexanet alfa was evaluated in Phase III ANNEXA (andexanet alfa, a novel antidote to anticoagulant effects of factor Xa inhibitors) which studies to test its safety and efficacy in healthy subjects anticoagulated with apixaban (ANNEXA A) and rivaroxaban (ANNEXA-R). However, the FDA is yet to approve andexanet alfa to reverse the effects of direct and indirect FXa inhibitors [15]. Ciraparantag/PER977, a small synthetic cationic molecule that binds direct FXa inhibitors, direct thrombin inhibitors, unfractionated heparin, low-molecular-weight heparin through noncovalent hydrogen bonds and charge-charge interactions, is in clinical development albeit it has the potential to cause platelet activation [7].

\section{New oral anticoagulants \& platelet aggregation inhibitors}

Platelet activation and platelet reactivity may pose a prothrombotic challenge in the management of patients who are receiving a combination therapy comprising antiplatelet and new oral anticoagulants and who require reversal agents to neutralize the anticoagulant effects. Patients on clopidogrel, a P2Y12 inhibitor which is activated by the CYP450 system and who carry the CYP2C19*2 allele, metabolize clopidogrel poorly and may be at a higher risk of thrombosis, especially when their concomitant new oral anticoagulant drugs are being antagonized by reversal agents for bleeding complications. Platelet hyper-reactivity may result in initial and recurrent arterial thrombosis [16]. Albeit newer P2Y12 inhibitors are increasingly becoming available, clopidogrel is still widely used. Voraxapar, a platelet PAR-1 antagonist was recently approved by the FDA. Common variants in the human platelet PAR-4 thrombin receptor are reported to alter platelet function and differ by race [17]. This could be significant in clinical scenarios where patients are concomitantly placed on voraxapar and new oral anticoagulant drugs, and require reversal agents to neutralize the anticoagulant effects.

Despite drug manufacturers' claims that a particular reversal agent does not have procoagulant effects upon neutralizing NOACs, there may still be chances of triggering of procoagulant effects due to the presence of any inherited or acquired thrombotic disorders. Since screening of patients for such disorders is not feasible each time a reversal agent is given, one cannot discount the possibility that a procoagulant or hypercoagulant response will not be triggered. The real world data on reversal agents will shed more light on the imminent adverse outcomes. Further- 
more, in some other scenarios where a combination of NOAC, antiplatelet and fibrinolytic is onboard, reversal of NOACs alone may not be sufficient and since the effects of other drugs are not counteracted, there could be adverse bleeding effects despite reversal agents. As these critical scenarios happen in emergency situations, these adverse outcomes may be unavoidable.

\section{Conclusion}

The concept of personalized medicine actually originated in India more than 5000 years ago, when Ayurveda, a natural healthcare system emphasized the treatment of disease in highly individualized manner and was best summed up at that time in a quotation by Charaka Samhita, "Every individual is different from another and hence should be considered as a different entity. As many variations are there in the universe, all are seen in human beings" [18].

All anticoagulant drugs have the potential to cause bleeding complications. In order to prevent bleeding or

\section{References}

1 Chan NC, Bhagirath V, Eikelboom JW. Profile of betrixaban and its potential in the prevention and treatment of venous thromboembolism. Vasc. Health Risk Manag. 11, 343-351 (2015).

2 Blann A. Nonvitamin K antagonist oral anticoagulants (NOACs): the tide continues to come in. Vasc. Health Risk Manag. 11, 489-492 (2015).

3 Connolly SJ, Ezekowitz MD, Yusuf S et al. Dabigatran versus warfarin in patients with atrial fibrillation. N. Engl. J. Med. 361(12), 1139-1151 (2009).

4 Granger CB, Alexander JH, McMurray JJ et al. Apixaban versus warfarin in patients with atrial fibrillation. N. Engl. J. Med. 365(11), 981-992 (2011).

5 Patel MR, Mahaffey KW, Garg J et al. Rivaroxaban versus warfarin in nonvalvular atrial fibrillation. N. Engl. J. Med. 365(10), 883-891 (2011).

6 Blann A. Nonvitamin K antagonist oral anticoagulants (NOACs): the tide continues to come in. Vasc. Health Risk Manag. 11, 489-492 (2015).

7 Hu TY, Vaidya VR, Asirvatham SJ. Reversing anticoagulant effects of novel oral anticoagulants: role of ciraparantag, andexanet alfa, and idarucizumab. Vasc. Health Risk Manag. 12, 35-44 (2016).

8 Sarode R, Milling TJ Jr, Refai MA et al. Efficacy and safety of a 4-factor prothrombin complex concentrate in patients on vitamin $\mathrm{k}$ antagonists presenting with major bleeding: a randomized, plasma controlled, Phase IIIb study. Circulation 128(11), 1234-1243 (2013).

9 Aronis KN, Hylek EM. Who, when, and how to reverse non-vitamin K oral anticoagulants. J. Thromb. Thrombolysis 41(2), 253-272 (2016). thrombotic complications, the doses of NOACs need to be calibrated or individualized in addition to the availability of better tests to effectively monitor them. Furthermore, in order to prevent thrombotic complications in patients who are on reversal agents to neutralize the anticoagulant effects of NOACs, the doses of reversal agents also need to be calibrated or individualized. In other words, the concepts of personalized or precision medicine need to be increasingly incorporated in the routine management of patients in order to achieve better clinical outcomes.

\section{Financial \& competing interests disclosure}

The authors have no relevant affiliations or financial involvement with any organization or entity with a financial interest in or financial conflict with the subject matter or materials discussed in the manuscript. This includes employment, consultancies, honoraria, stock ownership or options, expert testimony, grants or patents received or pending, or royalties.

No writing assistance was utilized in the production of this manuscript.

10 Marlu R, Hodaj E, Paris A, Albaladejo P, Cracowski JL, Pernod G. Effect of non-specific reversal agents on anticoagulant activity of dabigatran and rivaroxaban: a randomized crossover ex vivo study in healthy volunteers. Thromb. Haemost. 108(2), 217-224(2012).

11 Thorborg C, Horn EP, Mofid H, Langer F. Reversal of specific antidote, idarucizumab, of elevated dabigatran exposure in a patient with rectal perforation and paralytic ileus. $B r . J$. Anaesth. 117(3), 407-409 (2016).

12 Henderson RS Jr, Deshpande S, Williams B, Taylor BS, Tanaka KA. Idarucizumab for dabigatran reversal in emergency type-A aortic dissection. J. Cardiothorac. Vasc. Anesth. doi:10.1053/j.jvca.2016.04.027 (2016) (Epub ahead of print).

13 Alhashem HM, Avendano C, Hayes BD, Winters ME. Persistent life-threatening hemorrhage after administration of idarucizumab. Am. J. Emerg. Med. doi:10.1016/j. ajem.2016.06.096 (2016) (Epub ahead of print).

14 Marino KK, Santiago RA, Dew RB et al. Management of dabigatran-associated bleeding with two doses of idarucizumab. Pharmacotherapy 36(10), e160-e165 (2016).

15 GlobeNewswire. Portola pharmaceuticals receives complete response letter from FDA for Biologics Application for Andexanet Alfa (2016).

https://globenewswire.com

16 Bray PF. Platelet hyperreactivity: predictive and intrinsic properties. Hematol. Oncol. Clin. North Am. 21(4), 633-646 (2007).

17 Edelstein LC, Simon LM, Lindsay CR et al. Common variants in the human platelet PAR4 thrombin receptor alter platelet function and differ by race. Blood 124(23), 3450-3458 (2014).

18 Chatterjee B, Pancholi J. Prakriti-based medicine: a step towards personalized medicine. Ayu 32 (2), 141-146 (2011). 